\title{
Effets de l'enfouissement des semences d'Aethusa cynapium, Chenopodium album, Euphorbia exigua et Sinapis arvensis sur leur capacité germinative et leur levée au champ
}

La chronologie de levée des mauvaises herbes s'exprime, pour un stock semencier déterminé, en fonction des conditions climatiques annuelles et particulièrement celles qui succèdent au semis.

Des semences d'Aethusa cynapium L., Chenopodium album L., Euphorbia exigua L. et Sinapis arvensis L. ont été enfouies dans le sol à une profondeur de $20 \mathrm{~cm}$, puis à différentes époques de l'année (octobre, mars et mai) elles ont été : 1) extraites pour estimation de la capacité germinative des semences et de leur mortalité, 2) disposées à une profondeur de $1,5 \mathrm{~cm}$ dans le but de simuler le semis d'une culture ; l'évolution de la capacité germinative des semences et l'apparition des levées de plantules au champ sont observées sur une période de 6 mois.

Chez Aethusa cynapium, Chenopodium album et Euphorbia exigua, la capacité germinative des semences est toujours amélioré par l'enfouissement dans le sol. On observe une évolution cyclique de la capacité germinative avec un maximum de novembre à avril pour Aethusa et Euphorbia et de février à juin pour Chenopodium. Les levées au champ n'apparaissent que de mars à juin (mars à juillet pour Chenopodium). Le taux de germination et de levée des semences après simulation de travail du sol est très variable; les maxima observés sont de 37 p. 100 pour Aethusa, 13 p. 100 pour Euphorbia et 9 p. 100 pour Chenopodium. Les travaux simulés de mai sont défavorables à la germination et à lá levée d'Aethusa et d'Euphorbia.

Chez Sinapis arvensis, l'enfouissement des semences dans le sol favorise également la germination, mais le taux de levées des plantules est plus important et atteint $60 \mathrm{p}$. 100. Les levées apparaissent en novembre-décembre puis de mai à juillet.

A la suite de leur enfouissement à $20 \mathrm{~cm}$ durant 19 mois, la mortalité des semences est faible pour Aethusa, Euphorbia et Chenopodium; elle est plus importante pour Sinapis.

Mots clés additionnels : Mauvaises herbes, germination, chronologie des levées.

For a determinate weed seed bank, the distribution of seedling emergence depends on the annual climatic conditions and particularly on those at the time of sowing of the crop. Freshly collected seeds of Aethusa cynapium L., Chenopodium album L., Euphorbia exigua L. and Sinapis arvensis L. were buried in the soil to a depth of $20 \mathrm{~cm}$ in September. Then, at different times in the year (October, March and May) the sceds were : 1) extracted for determination of mortality in the soil and germinative ability, 2) buried at a depth of $1.5 \mathrm{~cm}$ in order to simulate soil cultivation before sowing of the crop, and the emerging seedlings were counted over 6 months. For Aethusa, Euphorbia and Chenopodium, the germinative ability of the seeds was always increased when buried in the soil and a cyclic change in this capacity was observed with a maximum from September to April for Aethusa and Euphorbia, from February to June for Chenopodium. The seedlings emerged in the field from March to June (July for Chenopodium). The percentage of viable seeds buried at a depth of $1.5 \mathrm{~cm}$ which gave rise to seedlings varied according to species and the date of simulated soil cultivation, and the maximum observed was $37 \%$ for Aethusa, $13 \%$ for Euphorbia and $9 \%$ for Chenopodium. Simulated soil cultivation in May was less favourable to germination and emergence for Aethusa and Euphorbia. For Sinapis arvensis, burying the seeds also increased the germinative ability but seedling emergence for this species was higher with a maximum of $60 \%$. Seedlings appeared in November-December and from May to July. After burial at $20 \mathrm{~cm}$ depth without soil cultivation for 19 months the mortality of seeds was very low for Aethusa, Euphorbia and Chenopodium, but higher for Sinapis. 


\section{INTRODUCTION}

L'échelonnement et la périodicité des levées de mauvaises herbes en culture est la résultante de l'aptitude à germer des semences et des conditions climatiques permettant ou non leur germination (JAUZEIN, 1986). Au champ, pour une même espèce, la capacité germinative des semences varie en fonction de la dormance primaire (innée) et de son évolution dans le sol. Fréquemment, des semences initialement dormantes, perdent cette inaptitude à germer après quelques mois d'enfouissement, mais les conditions pédoclimatiques de conservation (température, humidité, atmosphère gazeuse...) peuvent aussi induire des dormances secondaires (KARSSEN, 1982; ChADOEUFHANNEL, 1985).

Les travaux culturaux de mise en place des cultures sont évidemment favorables à la germination et à la levée des mauvaises herbes lorsque la température du sol correspond aux exigences germinatives spécifiques; un même stock semencier s'exprimera différemment en fonction de ce paramètre. Aussi, pour juger du comportement de 4 espèces de mauvaises herbes à l'implantation de différentes cultures, nous avons enfoui puis extrait du sol des semences d'Aethusa cynapium L. (petite ciguë), de Chenopodium album L. (chénopode blanc), d'Euphorbia exigua L. (petite euphorbe) et de Sinapis arvensis L. (moutarde des champs) à différentes époques correspondant à la mise en place d'une céréale d'hiver, d'une culture précoce (pois, orge) ou tardive (betterave, maïs) de printemps.

\section{MATÉRIEL ET MÉTHODE}

\section{A. Semences}

\section{Origine et conservation}

Les semences ont été récoltées à maturité dans des parcelles normalement cultivées du Domaine d'Epoisses (sol argilo-limoneux), en Côte-d'Or. Les dates de récoltes ainsi que les cultures sont répertoriées (tabl. 1).

TABLEAU 1

Origine et nombre de semences enfouies par cagette. Origin and number of huried seeds per tray.

\begin{tabular}{llrr}
\hline \hline Espèces & Cultures & Date de récolte & \multicolumn{1}{c}{$\begin{array}{c}\text { Nombre } \\
\text { de semences }\end{array}$} \\
\hline Aethusa cynapium & Betteraves & $3 / 9 / 82$ & $1500 \pm 29(1)$ \\
Chenopodium album & Betteraves & $25 / 8 / 82$ & $2000 \pm 24(1)$ \\
Euphorbia exigua & Betteraves & $23 / 8 / 82$ & $2000 \pm 132(1)$ \\
Sinapis arvensis & Tournesol & $27 / 7 / 82$ & 1500 \\
\hline \hline
\end{tabular}

(1) Estimation par pesée. Estimation by weighing

(2) Comptage. Counting.

Après la récolte, les semences sont placées dans des sachets de papier stockés au laboratoire. La capacité germinative des semences ainsi conservées est éprouvée pendant toute la durée de l'expérimentation par des tests de germination décrits ci-après.

\section{Enfouissement}

Des lots de semences sont constitués, suivant les espèces par comptage ou par pesée (tabl. 1). Peu avant leur enfouissement ces semences sont disposées sur un voile de tergal, lequel est placé dans une cagette en polyéthylène $(\mathrm{L}=40,1=20, \mathrm{H}=7 \mathrm{~cm})$ contenant de la terre du Domaine grossièrement tamisée, jusqu'à $1,5 \mathrm{~cm} \mathrm{du}$ bord. Le tergal et les semences sont ensuite recouverts de terre tamisée à $3,5 \mathrm{~mm}$.

L'enfouissement des cagettes ainsi préparées est effectué dans des tranchées de $30 \mathrm{~cm}$ de profondeur garnies de terre meuble, dans un champ non cultivé. Les cagettes sont ensuite recouvertes de terre de façon à ce que les semences soient situées à $20 \mathrm{~cm}$ de profondeur. L'enfouissement est réalisé suivant un dispositif de 4 blocs contenant chacun 24 cagettes nécessaires aux extractions successives.

\section{B. Etude de la conservation et de la capacité germinative des semences enfouies}

L'extraction des cagettes s'effectue en octobre, mars et mai, durant 2 années consécutives (1983-1984), aux dates précisées au tableau 2. Aussitôt après, le contenu de certaines cagettes est étudié en chambre noire munie d'un éclairage vert. Le voile de tergal est débarrassé de la terre et des semences qu'il contient sous filet d'eau et au-dessus d'un tamis à mailles de $0,6 \mathrm{~mm}$. Les semences sont alors extraites ; 200 d'entre elles, morphologiquement normales, sont utilisées pour la mise en place des tests de germination. Les semences restantes sont ensuite rapidement séchées puis comptées pour estimation des pertes durant la période d'enfouissement.

Les tests de germination sont effectués sur papier buvard imbibé d'eau déminéralisée, en bacs de germination de type Jacobsen, à raison de 100 semences par répétition. Ces bacs sont placés en enceinte climatique à l'alternance de température $15-10^{\circ} \mathrm{C}(12 \mathrm{~h}-12 \mathrm{~h})$, favorable à la germination de ces espèces, à l'obscurité totale (germinations à l'obscurité), ou en présence de lumière durant $12 \mathrm{~h}$ à $15^{\circ} \mathrm{C}$ (germinations à la lumière). La source lumineuse est constituée de tubes fluorescents type Blanc Industrie Philips et de lampes à incandescence, le tout dispersant une énergie de $34 \mathrm{~W} \cdot \mathrm{cm}^{2}$. Les comptages de germination sont réalisés 2 à 3 fois par semaine durant 21 jours, en chambre noire et sous éclairage inactinique vert pour les tests réalisés à l'obscurité. Les semences non germées sont alors disposées dans de nouveaux bacs de germination contenant $250 \mathrm{ml}$ d'une solution d'acide gibbérellique (GA3) à $1 \mathrm{~g} / 1$ dont $20 \mathrm{ml}$ sont pulvérisés directement sur les semences. Les bacs sont replacés dans les mêmes conditions pour une nouvelle période de 21 jours.

\section{Etude des levées au champ et du stock en semences}

En même temps que sont réalisées les extractions pour l'étude qualitative et quantitative des semences, d'autres 
cagettes sont disposées sur le champ de manière à ce que leur bord supérieur affleure le niveau du sol; les semences se situent alors à une profondeur théorique de 1,5 à $2 \mathrm{~cm}$; on simule ainsi une perturbation du sol (de type labour) permettant aux semences d'être enfouies à une profondeur favorable à leur germination et à leur levée en l'absence de lumière. Après chaque positionnement superficiel des cagettes (octobre, mars et mai), on dénombre les levées de plantules apparues au cours d'une période de 1, 3 et 6 mois ; lors des comptages, les plantules sont sectionnées ou délicatement arrachées de façon à perturber le moins possible la surface du sol. A l'issue de ces périodes d'observation, les cagettes sont retirées du champ et leur contenu est étudié par les méthodes décrites en $\mathrm{B}$ ) : comptage et tests de germination.

\section{Analyse et expression des résultats}

Les tests de germination sont exprimés en p. 100 de germination, sur eau ou sur eau puis GA3. L'analyse statistique de ces valeurs est réalisée par l'analyse de variance à 4 répétitions, après transformation des données (arcsin Rac. p. 100). La comparaison des moyennes est faite par le test de Newman et Keuls au risque de 5 p. 100 .

Les estimations de disparition de semences durant l'enfouissement à $20 \mathrm{~cm}$ et à 1,5-2 cm font aussi l'objet d'une analyse de variance, bien que la précision des observations soit mauvaise (coefficients de variation compris entre 3 et 28 p. 100 pour Aethusa cynapium, 7 et 17 p. 100 pour Chenopodium album, 7 et 18 p. 100 pour Euphorbia exigua, 9 et 28 p. 100 pour Sinapis arvensis). Par contre, les comptages effectués sur les levées de plantules au champ ne permettent pas une analyse statistique car plusieurs cagettes ont subi une dessiccation trop importante et ont dû être éliminées.

\section{RÉSULTATS}

\section{A. Capacité germinative des semences conservées au sec}

Aethusa cynapium (tabl. 2A) : les semences sèches ne germent pas sur eau, ni à la lumière, ni à l'obscurité, alors qu'à la récolte, les tests réalisés en présence d'acide gibbérellique permettent d'observer une viabilité des semences voisine de 80 p. 100 . Au cours de la conservation, le taux de germination diminue, moins en présence de lumière qu'à l'obscurité. Ceci suggère que la postmaturation des akènes au sec instaure une dormance observable en particulier à l'obscurité.

Euphorbia exigua (tabl. 2B) : la dormance des semences est importante mais évolue dans le temps : 3,5 p. 100 de germination à la récolte et 14 p. 100 après 2 ans de conservation (moyennes des tests réalisés sur eau, à la lumière et à l'obscurité). L'action de l'acide gibbérellique est relativement faible sur cette espèce ( 45 p. 100 de germination en moyenne), cependant différentes observations effectuées au laboratoire sur l'efficacité de

\section{TABLEAU 2}

Evolution de la capacité germinative exprimée en $p .100)$, des semences d'Aethusa cynapium $(A), d$ 'Euphorbia exigua $(B)$, de Chenopodium album $(C)$ et de Sinapis arvensis $(D)$, conservées au sec ou extraites du sol aux dates E1,E2, ... E6. Les tests de germination sont réalisés en enceintes climatiques à alternance $15-10^{\circ} \mathrm{C}(12 \mathrm{~h}-12 \mathrm{~h})$ en présence de lumière $\left(12 \mathrm{~h}\right.$ a $\left.15^{\circ} \mathrm{C}\right)$ ou à l'obscurité sur eau $\left(\mathrm{H}_{2} \mathrm{O}\right)$ puis sur acide gibbérellique $\left(\mathrm{GA} \mathrm{A}_{3}\right)$. (a), (b), ... indiquent des valeurs significativement différentes au seuil de $5 \%$ pour des conditions de conservation identiques.

Germinative ability (as \%) of seeds of Aethusa cynapium (A), Euphorbia exigua (B). Chenopodium album (C) and Sinapis arvensis $(D)$ after $d r y$ or buried storage at the dates E1, E2, .. E6. Tests were made in water $\left(\mathrm{H}_{2} \mathrm{O}\right)$ or gibberellic acid $\left(\mathrm{GA}_{3}\right)$ in incuhators at $15-10^{\circ} \mathrm{C}$ alternating temperature $(12 \mathrm{~h}-\mathrm{I} 2 \mathrm{~h})$ with $12 \mathrm{~h}$ at $15^{\circ} \mathrm{C}$ photoperiod or in the dark. (a), (b), ... indicates significant values ( $5 \%$ ) for the same condition of storage.

(A) Aethusa cynapium : Enfouissement le 10/9/82

\begin{tabular}{|c|c|c|c|c|c|c|c|c|}
\hline \multirow{3}{*}{ Dates } & \multicolumn{4}{|c|}{ Conservation au sec } & \multicolumn{4}{|c|}{ Conservation à $-20 \mathrm{~cm}$} \\
\hline & \multicolumn{2}{|c|}{ Lum. } & \multicolumn{2}{|c|}{ Obsc. } & \multicolumn{2}{|c|}{ Lum. } & \multicolumn{2}{|c|}{ Obsc. } \\
\hline & $\mathrm{H}_{2} \mathrm{O}$ & GA3 & $\mathrm{H}_{2} \mathrm{O}$ & GA3 & $\mathrm{H}_{2} \mathrm{O}$ & GA3 & $\mathrm{H}_{2} \mathrm{O}$ & GA3 \\
\hline $10 / 82(\mathrm{E} 1)$ & - & 一 & - & - & $\begin{array}{c}5 \\
(\mathrm{gh})\end{array}$ & $\begin{array}{c}41 \\
\text { (cd) }\end{array}$ & $\begin{array}{r}0 \\
(\mathrm{~h})\end{array}$ & $\begin{array}{c}24 \\
\text { (defg) }\end{array}$ \\
\hline $11 / 82$ & $\begin{array}{c}0 \\
\text { (f) }\end{array}$ & $\begin{array}{l}79 \\
\text { (a) }\end{array}$ & $\begin{array}{r}0 \\
(f)\end{array}$ & $\begin{array}{l}83 \\
\text { (a) }\end{array}$ & - & - & - & - \\
\hline $3 / 83(\mathrm{E} 2)$ & - & - & - & - & $\begin{array}{c}33 \\
\text { (cde) }\end{array}$ & $\begin{array}{l}67 \\
\text { (b) }\end{array}$ & $\begin{array}{c}\text { l } \\
\text { (h) }\end{array}$ & $\begin{array}{c}25 \\
(\mathrm{def})\end{array}$ \\
\hline $4 / 83$ & $\begin{array}{c}0 \\
(f)\end{array}$ & $\begin{array}{l}41 \\
\text { (d) }\end{array}$ & $\begin{array}{c}0 \\
\text { (f) }\end{array}$ & $\begin{array}{r}7 \\
\text { (f) }\end{array}$ & - & - & - & - \\
\hline $5 / 83(\mathrm{E} 3)$ & - & - & - & - & $\begin{array}{c}41 \\
(\mathrm{~cd})\end{array}$ & $\begin{array}{l}59 \\
\text { (b) }\end{array}$ & $\begin{array}{r}2 \\
\text { (h) }\end{array}$ & $\begin{array}{c}13 \\
(\mathrm{fgh})\end{array}$ \\
\hline $10 / 83(\mathrm{E} 4)$ & - & - & - & - & $\begin{array}{l}97 \\
\text { (a) }\end{array}$ & $\begin{array}{l}99 \\
\text { (a) }\end{array}$ & $\begin{array}{c}20 \\
\text { (fgh) }\end{array}$ & $\begin{array}{c}49 \\
\text { (bc) }\end{array}$ \\
\hline $12 / 83$ & $\begin{array}{c}1 \\
(f)\end{array}$ & $\begin{array}{l}62 \\
\text { (b) }\end{array}$ & $\begin{array}{r}0 \\
\text { (f) }\end{array}$ & $\begin{array}{l}16 \\
\text { (e) }\end{array}$ & - & - & - & - \\
\hline $3 / 84$ (E5) & - & - & - & - & $\begin{array}{l}96 \\
\text { (a) }\end{array}$ & $\begin{array}{l}98 \\
\text { (a) }\end{array}$ & $\begin{array}{l}91 \\
\text { (a) }\end{array}$ & $\begin{array}{l}95 \\
\text { (a) }\end{array}$ \\
\hline $5 / 84(\mathrm{E} 6)$ & - & - & 一 & - & $\begin{array}{l}64 \\
\text { (b) }\end{array}$ & $\begin{array}{l}66 \\
\text { (b) }\end{array}$ & $\begin{array}{c}10 \\
(\mathrm{fgh})\end{array}$ & $\begin{array}{c}11 \\
(\mathrm{fgh})\end{array}$ \\
\hline $10 / 84$ & $\begin{array}{c}1 \\
\text { (f) }\end{array}$ & $\begin{array}{l}56 \\
\text { (c) }\end{array}$ & $\begin{array}{c}0 \\
(f)\end{array}$ & $\begin{array}{r}4 \\
\text { (f) }\end{array}$ & - & - & - & - \\
\hline
\end{tabular}


(B) Euphorbia exigua : Enfouissement le 10/9/82.

\begin{tabular}{|c|c|c|c|c|c|c|c|c|}
\hline \multirow{3}{*}{ Dates } & \multicolumn{4}{|c|}{ Conservation au sec } & \multicolumn{4}{|c|}{ Conservation à $-20 \mathrm{~cm}$} \\
\hline & \multicolumn{2}{|c|}{ Lum. } & \multicolumn{2}{|c|}{ Obsc. } & \multicolumn{2}{|c|}{ Lum. } & \multicolumn{2}{|c|}{ Obsc. } \\
\hline & $\mathrm{H}_{2} \mathrm{O}$ & GA3 & $\mathrm{H}_{2} \mathrm{O}$ & $\mathrm{GA} 3$ & $\mathrm{H}_{2} \mathrm{O}$ & GA3 & $\mathrm{H}_{2} \mathrm{O}$ & GA3 \\
\hline $10 / 82(\mathrm{E} 1)$ & - & - & - & - & $\stackrel{1}{(h)}$ & $\begin{array}{l}60 \\
\text { (b) }\end{array}$ & $\begin{array}{r}2 \\
(\mathrm{~h})\end{array}$ & $\begin{array}{l}65 \\
\text { (b) }\end{array}$ \\
\hline $11 / 82$ & $\begin{array}{r}5 \\
(\mathrm{ef})\end{array}$ & $\begin{array}{l}52 \\
\text { (b) }\end{array}$ & $\begin{array}{r}2 \\
(f)\end{array}$ & $\begin{array}{c}50 \\
\text { (b) }\end{array}$ & & & & \\
\hline $3 / 82(\mathrm{E} 2)$ & - & - & - & - & $\begin{array}{c}1 \\
\text { (h) }\end{array}$ & $\begin{array}{c}27 \\
\text { (def) }\end{array}$ & $\begin{array}{c}20 \\
(\mathrm{ef})\end{array}$ & $\begin{array}{c}65 \\
\text { (b) }\end{array}$ \\
\hline $4 / 83$ & $\begin{array}{r}7 \\
(\mathrm{ef})\end{array}$ & $\begin{array}{c}60 \\
(\mathrm{ab})\end{array}$ & $\begin{array}{c}9 \\
(\mathrm{ef})\end{array}$ & $\begin{array}{l}65 \\
\text { (a) }\end{array}$ & - & $\cdots$ & - & - \\
\hline $5 / 83(\mathrm{E} 3)$ & - & - & - & - & $\begin{array}{r}0 \\
(\mathrm{~h})\end{array}$ & $\begin{array}{l}55 \\
\text { (b) }\end{array}$ & $\begin{array}{c}8 \\
(\mathrm{gh})\end{array}$ & $\begin{array}{c}58 \\
\text { (b) }\end{array}$ \\
\hline $10 / 83(\mathrm{E} 4)$ & - & 一 & 一. & - & $\begin{array}{c}31 \\
\text { (de) }\end{array}$ & $\begin{array}{l}62 \\
\text { (b) }\end{array}$ & $\begin{array}{c}23 \\
(\operatorname{def})\end{array}$ & $\begin{array}{l}76 \\
\text { (a) }\end{array}$ \\
\hline $12 / 83$ & $\begin{array}{r}14 \\
\text { (e) }\end{array}$ & $\begin{array}{c}26 \\
\text { (cd) }\end{array}$ & $\begin{array}{c}10 \\
(\mathrm{ef})\end{array}$ & $\begin{array}{c}26 \\
\text { (cd) }\end{array}$ & - & - & - & - \\
\hline $3 / 84(\mathrm{E} 5)$ & - & - & - & $\cdots$ & $\begin{array}{c}15 \\
(\mathrm{fg})\end{array}$ & $\begin{array}{c}60 \\
\text { (b) }\end{array}$ & $\begin{array}{c}35 \\
(\mathrm{~cd})\end{array}$ & $\begin{array}{l}58 \\
\text { (b) }\end{array}$ \\
\hline $5 / 84(\mathrm{E} 6)$ & - & - & - & - & $\begin{array}{c}8 \\
(\mathrm{gh})\end{array}$ & $\begin{array}{c}24 \\
\text { (def) }\end{array}$ & $\begin{array}{c}7 \\
(\mathrm{gh})\end{array}$ & $\begin{array}{l}42 \\
\text { (c) }\end{array}$ \\
\hline $10 / 84$ & $\begin{array}{l}22 \\
\text { (d) }\end{array}$ & $\begin{array}{l}50 \\
\text { (b) }\end{array}$ & $\begin{array}{c}7 \\
\text { (cf) }\end{array}$ & $\begin{array}{l}34 \\
\text { (c) }\end{array}$ & - & $\ldots$ & - & - \\
\hline
\end{tabular}

GA3 sur cette espèce, ont montré que des concentrations supérieures à $1 \mathrm{~g} / 1$ (concentration déjà très élevée) augmentaient encore le taux de germination. Les potentialités germinatives de la petite euphorbe sont donc ici sous-estimées.

Chenopodium album (tabl. 2C): les semences manifestent une dormance importante à la récolte et ne germent sur eau qu'en présence de lumière (17 p. 100 de germination). Les traitements à l'acide gibbérellique améliorent la germination $(41$ p. 100 à la lumière et 22 p. 100 à l'obscurité) et permettent d'observer une baisse de la capacité germinative des semences au cours de leur conservation au sec. Les traitements effectués à l'obscurité ont une efficacité fluctuante qui rend compte du mauvais état des semences (moisissures) après 21 jours de mise en germination sur eau.

Sinapis arvensis (tabl. 2D): la dormance initialement très importante des graines à la récolte est éliminée partiellement avec le temps pour atteindre 31 p. 100 de germination à la lumière au bout de 2 ans de conservation et 14 p. 100 à l'obscurité après 1 an. Les traitements avec GA3, qui permettent de lever complètement la dormance des graines à la récolte, sont d'autant moins efficaces que les semences vieillissent ; la photosensibilité positive subsiste.

(C) Chenopodium album : Enfouissement le 10/9/82.

\begin{tabular}{|c|c|c|c|c|c|c|c|c|}
\hline \multirow{3}{*}{ Dates } & \multicolumn{4}{|c|}{ Conservation au sec } & \multicolumn{4}{|c|}{ Conservation $\grave{a}-20 \mathrm{~cm}$} \\
\hline & \multicolumn{2}{|c|}{ Lum. } & \multicolumn{2}{|c|}{ Obsc. } & \multicolumn{2}{|c|}{ Lum. } & \multicolumn{2}{|c|}{ Obsc. } \\
\hline & $\mathrm{H}_{2} \mathrm{O}$ & GA3 & $\mathrm{H}_{2} \mathrm{O}$ & GA3 & $\mathrm{H}_{2} \mathrm{O}$ & GA3 & $\mathrm{H}_{2} \mathrm{O}$ & GA3 \\
\hline $10 / 82(\mathrm{E} 1)$ & - & - & - & - & $\begin{array}{l}21 \\
\text { (d) }\end{array}$ & $\begin{array}{l}23 \\
\text { (d) }\end{array}$ & $\begin{array}{r}0 \\
\text { (e) }\end{array}$ & $\begin{array}{c}2 \\
\text { (e) }\end{array}$ \\
\hline $11 / 82$ & $\begin{array}{c}17 \\
(\mathrm{~cd})\end{array}$ & $\begin{array}{l}41 \\
\text { (a) }\end{array}$ & $\begin{array}{r}0 \\
\text { (f) }\end{array}$ & $\begin{array}{l}22 \\
\text { (b) }\end{array}$ & - & - & - & - \\
\hline $3 / 83(\mathrm{E} 2)$ & - & - & - & - & $\begin{array}{l}88 \\
\text { (a) }\end{array}$ & $\begin{array}{l}89 \\
\text { (a) }\end{array}$ & $\begin{array}{c}78 \\
(\mathrm{ab})\end{array}$ & $\begin{array}{c}79 \\
(\mathrm{ab})\end{array}$ \\
\hline $4 / 83$ & $\begin{array}{c}17 \\
\text { (cd) }\end{array}$ & $\begin{array}{l}40 \\
\text { (a) }\end{array}$ & $\begin{array}{r}0 \\
\text { (f) }\end{array}$ & $\begin{array}{c}13 \\
\text { (cde) }\end{array}$ & - & - & - & - \\
\hline $5 / 83(\mathrm{E} 3)$ & - & - & - & - & $\begin{array}{c}72 \\
(\mathrm{ab})\end{array}$ & $\begin{array}{c}71 \\
(\mathrm{ab})\end{array}$ & $\begin{array}{l}58 \\
\text { (b) }\end{array}$ & $\begin{array}{l}59 \\
\text { (b) }\end{array}$ \\
\hline $10 / 83(\mathrm{E} 4)$ & - & - & - & - & $\begin{array}{r}6 \\
\text { (e) }\end{array}$ & $\begin{array}{r}7 \\
\text { (e) }\end{array}$ & $\begin{array}{r}3 \\
\text { (e) }\end{array}$ & $\begin{array}{r}4 \\
\text { (e) }\end{array}$ \\
\hline $12 / 83$ & $\begin{array}{c}8 \\
\text { (def) }\end{array}$ & $\begin{array}{c}19 \\
(\mathrm{bc})\end{array}$ & $\begin{array}{r}2 \\
\text { (f) }\end{array}$ & $\begin{array}{c}12 \\
\text { (cde) }\end{array}$ & - & - & - & - \\
\hline 3/84 (E5) & - & - & - & $\ldots$ & $\begin{array}{c}83 \\
(\mathrm{ab})\end{array}$ & $\begin{array}{c}84 \\
(a b)\end{array}$ & $\begin{array}{l}42 \\
\text { (c) }\end{array}$ & $\begin{array}{l}44 \\
\text { (c) }\end{array}$ \\
\hline $5 / 84(\mathrm{E} 6)$ & - & - & - & $\cdots$ & $\begin{array}{c}64 \\
(\mathrm{ab})\end{array}$ & $\begin{array}{c}66 \\
(\mathrm{ab})\end{array}$ & $\begin{array}{l}10 \\
\text { (c) }\end{array}$ & $\begin{array}{l}11 \\
\text { (e) }\end{array}$ \\
\hline $10 / 84$ & $\begin{array}{c}11 \\
\text { (cde) }\end{array}$ & $\begin{array}{l}23 \\
\text { (b) }\end{array}$ & $\begin{array}{r}0 \\
(\text { (f) }\end{array}$ & $\begin{array}{r}6 \\
(\mathrm{ef})\end{array}$ & - & - & - & - \\
\hline
\end{tabular}


(D) Sinapis arvensis : Enfouissement le 24/8/82.

\begin{tabular}{|c|c|c|c|c|c|c|c|c|}
\hline \multirow{3}{*}{ Dates } & \multicolumn{4}{|c|}{ Conservation au sec } & \multicolumn{4}{|c|}{ Conservation à $-20 \mathrm{~cm}$} \\
\hline & \multicolumn{2}{|c|}{ Lum. } & \multicolumn{2}{|c|}{ Obsc. } & \multicolumn{2}{|c|}{ Lum. } & \multicolumn{2}{|c|}{ Obsc. } \\
\hline & $\mathrm{H} 20$ & GA3 & $\mathrm{H} 20$ & GA3 & $\mathrm{H} 20$ & GA3 & $\mathbf{H} 20$ & GA3 \\
\hline $10 / 82(\mathrm{E} 1)$ & - & - & 一 & - & $\begin{array}{r}4 \\
(\mathrm{e})\end{array}$ & $\begin{array}{l}99 \\
\text { (a) }\end{array}$ & $\begin{array}{r}6 \\
\text { (e) }\end{array}$ & $\begin{array}{c}95 \\
(\mathrm{ab})\end{array}$ \\
\hline $11 / 82$ & $\begin{array}{c}1 \\
(\mathrm{~h})\end{array}$ & $\begin{array}{l}99 \\
\text { (a) }\end{array}$ & $\begin{array}{r}0 \\
(\mathrm{~h})\end{array}$ & $\begin{array}{l}97 \\
(a)\end{array}$ & - & - & - & - \\
\hline $3 / 83(\mathrm{E} 2)$ & - & - & - & - & $\begin{array}{c}68 \\
(\mathrm{~cd})\end{array}$ & $\begin{array}{c}95 \\
(\mathrm{ab})\end{array}$ & $\begin{array}{l}75 \\
\text { (c) }\end{array}$ & $\begin{array}{c}95 \\
\text { (ab) }\end{array}$ \\
\hline $4 / 83$ & $\begin{array}{l}18 \\
(\mathrm{~g})\end{array}$ & $\begin{array}{c}88 \\
\text { (bc) }\end{array}$ & $\begin{array}{c}9 \\
\text { (gh) }\end{array}$ & $\begin{array}{c}75 \\
\text { (de) }\end{array}$ & - & - & - & - \\
\hline $5 / 83(\mathrm{E} 3)$ & - & - & - & - & $\begin{array}{c}83 \\
(a b c)\end{array}$ & $\begin{array}{r}100 \\
(\mathrm{a})\end{array}$ & $\begin{array}{l}80 \\
(c)\end{array}$ & $\begin{array}{r}100 \\
(\mathrm{a})\end{array}$ \\
\hline $10 / 83(\mathrm{E} 4)$ & - & - & - & - & $\begin{array}{c}66 \\
\text { (cd) }\end{array}$ & $\begin{array}{c}88 \\
(a b c)\end{array}$ & $\begin{array}{l}59 \\
\text { (d) }\end{array}$ & $\begin{array}{c}91 \\
(\mathrm{ab})\end{array}$ \\
\hline $12 / 83$ & $\begin{array}{l}13 \\
(g)\end{array}$ & $\begin{array}{c}93 \\
(a b)\end{array}$ & $\begin{array}{l}14 \\
(\mathrm{~g})\end{array}$ & $\begin{array}{c}80 \\
\text { (cd) }\end{array}$ & - & -- & - & - \\
\hline $3 / 84(\mathrm{E} 5)$ & - & - & - & - & $\begin{array}{c}71 \\
\text { (cd) }\end{array}$ & $\begin{array}{c}81 \\
(a b c)\end{array}$ & $\begin{array}{l}77 \\
\text { (c) }\end{array}$ & $\begin{array}{c}82 \\
(a b c)\end{array}$ \\
\hline $5 / 84(\mathrm{E} 6)$ & - & - & - & - & $\begin{array}{r}53 \\
\text { (d) }\end{array}$ & $\begin{array}{c}64 \\
\text { (cd) }\end{array}$ & $\begin{array}{c}66 \\
\text { (cd) }\end{array}$ & $\begin{array}{c}74 \\
\text { (cd) }\end{array}$ \\
\hline $10 / 84$ & $\begin{array}{l}31 \\
(f)\end{array}$ & $\begin{array}{c}86 \\
\text { (bc) }\end{array}$ & $\begin{array}{c}9 \\
\text { (gh) }\end{array}$ & $\begin{array}{l}69 \\
\text { (e) }\end{array}$ & 一 & - & - & - \\
\hline
\end{tabular}

\section{B. Effets de l'enfouissement profond sur la capacité de germination des semences}

Chez les 4 espèces on observe une augmentation plus ou moins importante et rapide de la capacité germinative, tant à l'obscurité qu'en présence de lumière.

Aethusa cynapium (tabl. 2A et fig. 1A : E1 0, E2 0,... E6 0) : le taux de germination croît régulièrement à la lumière. A l'obscurité, l'effet de stratification de l'hiver 83 a été inefficace, la capacité germinative est faible mais atteint un maximum (91 p. 100) à la suite de l'extraction de mars 84 puis diminue (10 p. 100) en mai; GA3 ne permet pas d'améliorer cette valeur.

Euphorbia exigua (tabl. 2B et fig. 1B : E1 0, E2 0,... E6 0): l'enfouissement ne modifie pas sensiblement la capacité germinative des semences en présence de lumière, il semble par contre favoriser leur germination à l'obscurité. En effet, si la dormance reste encore importante (maximum: 35 p. 100), on constate d'une part une augmentation de la capacité germinative avec la durée d'enfouissement, et d'autre part, comme pour Aethusa à l'obscurité, des maxima de germination à la suite des extractions de mars.

Chenopodium album (tabl. 2C et fig. 1C: E1 0, E2 0, ... E6 0) : contrairement aux deux espèces précédentes, les effets de l'enfouissement sont équivalents en 82-83 et 83-84, à la suite des extractions de mars, à la lumière ou à l'obscurité. La stratification hivernale est efficace dès la première année. L'acide gibbérellique est sans action sur les semences préalablement enfouies, tant à la lumière qu'à l'obscurité, alors qu'il avait une efficacité faible mais significative sur semences sèches. Dans ce cas, l'enfouissement joue le même rôle que GA3 dans l'aptitude à lever une dormance partielle. Les taux de germination d'octobre sont plus faibles qu'en mars et mai.

Sinapis arvensis (tabl. 2D et fig. 1D : E1 0, E2 0, .. E6 0) : les semences initialement fortement dormantes (dormance levée entièrement par GA3) sont capables de germer abondamment dès le printemps. En deuxième année on assiste à une baisse de la capacité germinative observable lors des tests sur eau et sur acide gibbérellique; cette diminution de l'aptitude à germer n'est pas toujours significative du fait du manque de précision de l'expérimentation pour cette espèce. La légère photosensibilité des semences sèches n’apparaît plus à la suite de l'enfouissement.

\section{Effets de l'enfouissement superficiel succédant à un enfouissement profond}

Les modifications de la capacité germinative des semences à la suite de leur positionnement à $1,5-2 \mathrm{~cm}$ de profondeur ne peuvent être analysées que dans la mesure où le stock en semences n'a pas été sensiblement diminué par suite des germinations qui modifient la composition initiale des lots de semences; ce qui est le cas pour la petite euphorbe et le chénopode blanc.

Euphorbia exigua (fig. 1B : E1 1, 3, 6, E2 1, 3, 6, ... E6 1, 3, 6): les semences germent systématiquement mieux à l'obscurité qu'à la lumière, ce qui confirme les observations consécutives aux enfouissements profonds. Les possibilités de germination dans le sol, c'est-à-dire la capacité de germination à l'obscurité, évoluent en fonction de 2 variables : la durée pendant laquelle les semences ont été enfouies profondément (le taux de germination est plus élevé en $2^{\mathrm{c}}$ année qu'en première), et l'époque d'observation (la capacité germinative est meilleure entre les mois de janvier et mai).

Chenopodium album (fig. 1C: E1 1, 3, 6, E2 1, 3, 6, ... E6 1,3,6) : à l'inverse de la petite euphorbe, la capacité germinative est plus élevée la $1^{\text {re }}$ année d'enfouissement. L'époque où les possibilités germinatives sont les plus importantes se situe entre les mois de janvier et juin; le minimum se situant en octobre-novembre, ce qui confirme les observations faites à la suite des enfouissements profonds (tabl. 2C). 


\section{A: Athusa cynapium}

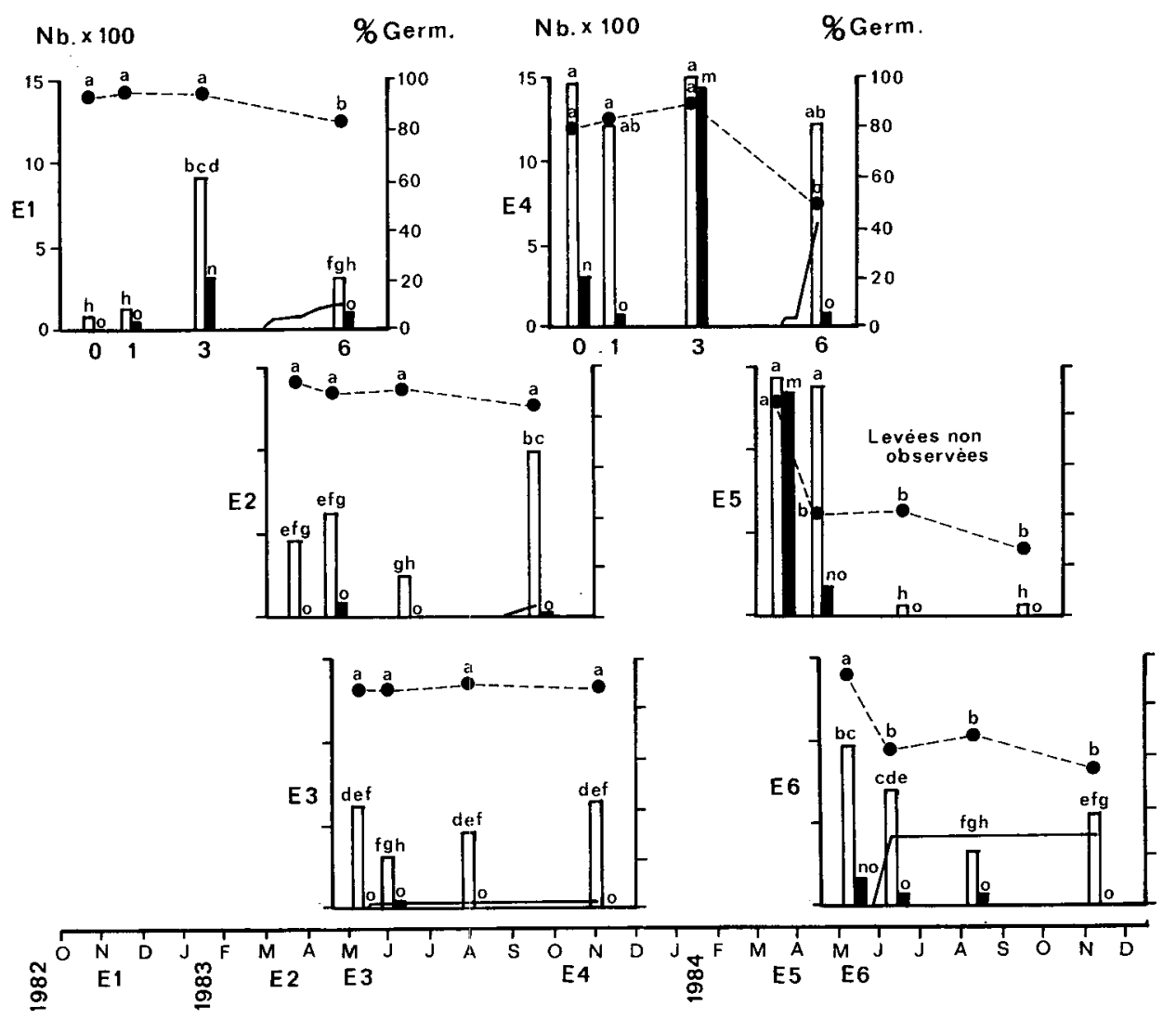

Figure 1

Evolution du stock en semences (-0-), des levées de plantules (___ ) et de la capacité germinative des semences testée à alternance de température $15-10^{\circ} \mathrm{C}(12 \mathrm{~h}-12 \mathrm{~h})$ en présence de $12 \mathrm{~h}$ de lumière $(\square)$ ou à l'obscurité ( $\left.\square\right)$, en fonction des dates d'extraction (E) puis de la mise en condition de germination de plein champ durant 1,3 et 6 mois, chez: Aethusa cynapium ( $A$ ), Euphorbia exigua ( $B$ ), Chenopodium album (C) et Sinapis arvensis (D) $, a, b, \ldots m, n, \ldots$ indiquent des valeurs significativement différentes au seuil de 5 p. 100 dans la comparaison des levées après chaque extraction et pour l'ensemble des valeurs de la capacité germinative.

Seed bank (-) , seedling emergence (—) and germinative ability at $15-10^{\circ} \mathrm{C}$ alternating temperature with $12 \mathrm{~h}$ photoperiod ( $\square$ ) or in the dark (D) according to extraction date (E) and seedling emergence period, during 1,3 and 6 months for: Aethusa cynapium ( $A$ ), Euphorbia exigua ( $B$ ), Chenopodium album $(C)$ and Sinapis arvensis $(D), a, b, \ldots m, n, \ldots$ indicate significant values ( $5 \%)$ in the comparison of seedling emergence after each extraction and for all values of germinative ability.

Chez ces 2 espèces ainsi que pour Aethusa cynapium la capacité germinative est toujours faible après un séjour prolongé (6 mois) des semences à faible profondeur. La capacité germinative des semences d'Aethusa est réduite durant les mois d'été.

\section{Chronologie des levées au champ et évolution du stock en semences (fig. 1)}

Aethusa cynapium (fig. 1A): les levées apparaissent exclusivement d'avril à juin. La première année cependant, les seules levées observées sont apparues dans les cagettes extraites en octobre. La capacité germinative des semences étant faible à l'obscurité, la mise en situation de germination des akènes pendant l'hiver à $1,5-2 \mathrm{~cm}$ a permis une meilleure stratification qu'à $20 \mathrm{~cm}$. En deuxième année, la capacité germinative a augmenté, permettant aux akènes de germer dès avril pour l'extraction d'automne, mais aussi dans le mois qui a suivi les extractions de mars et mai.

Euphorbia exigua (fig. 1B) : le nombre des levées pour cette espèce est faible, dans le meilleur des cas (extrac- tion d'octobre 1982) il ne dépasse pas 13 p. 100 des semences enfouies. Comme pour Aethusa, les levées apparaissent d'avril à début juin; les causes de nongermination sont cependant différentes : si les akènes de petite ciguë sont incapables de germer en fin de printemps du fait de leur importante dormance à l'obscurité, les graines d'euphorbe possèdent une capacité germinative qui leur permettrait de germer, mais la température du sol à cette époque de l’année est trop élevée pour permettre la germination.

Chenopodium album (fig. 1C): les levées, exprimées par rapport au stock initial, sont comprises entre 0,1 et 3,7 p. 100 (en avril et mai) pour les extractions d'octobre et de mars. En juillet-septembre 1983 une $2^{\mathrm{e}}$ levée permet d'atteindre 7 p. 100. La levée de plantules est donc très faible et s'échelonne d'avril à septembre, époque où la capacité germinative des akènes est faible. Les semences se conservent relativement bien dans le sol : le taux de disparition, compte tenu des levées, est au maximum de 21 p. 100 après 2 ans d'enfouissement (dont' 6 mois à $1,5-2 \mathrm{~cm}$ ).

Sinapis arvensis (fig. 1D) : les levées sont observées en mars dès la $1^{\text {re }}$ année pour les extractions d'octobre: 


\section{B: Euphorbia exigua}
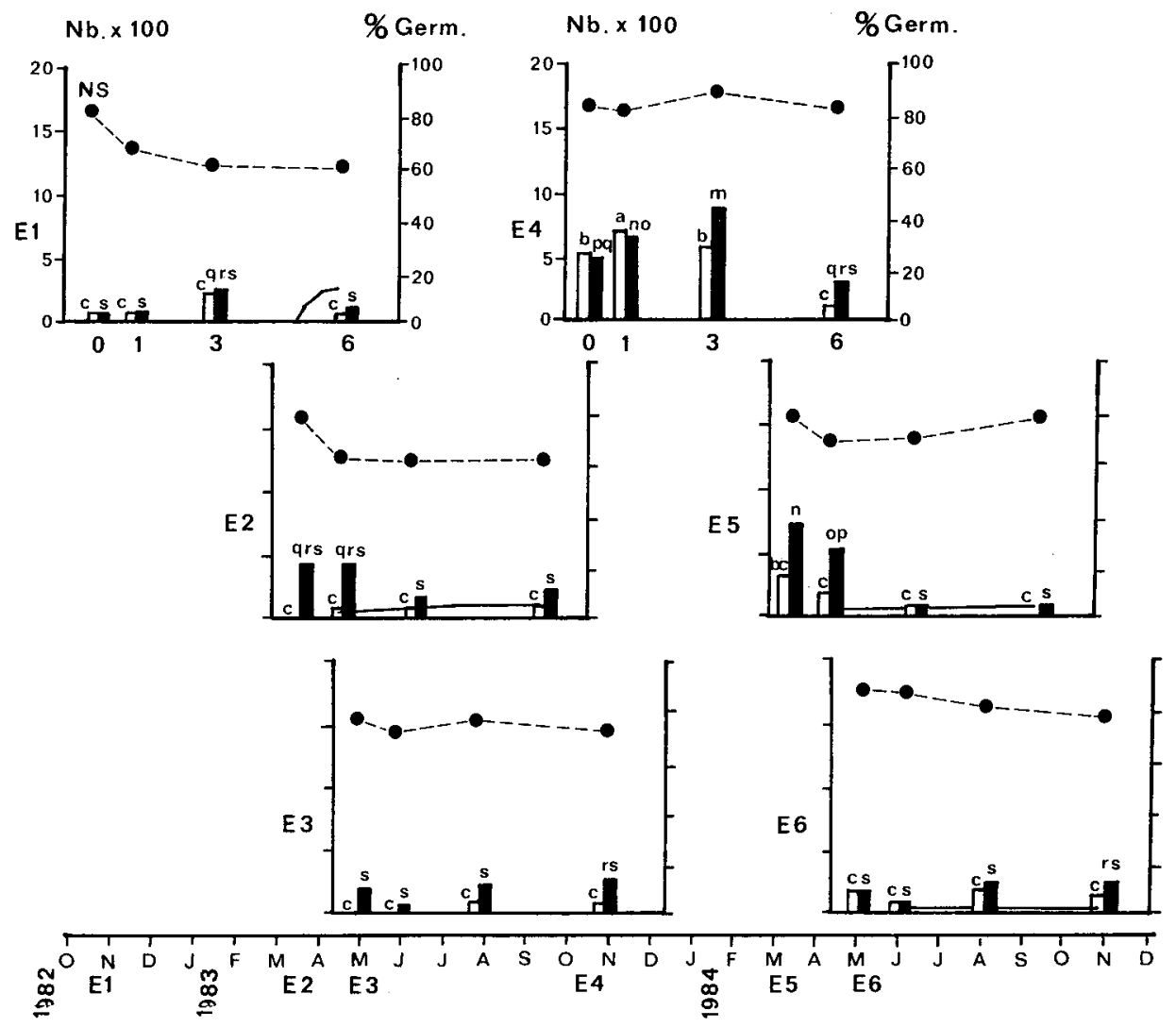

\section{C: Chenopodium album}
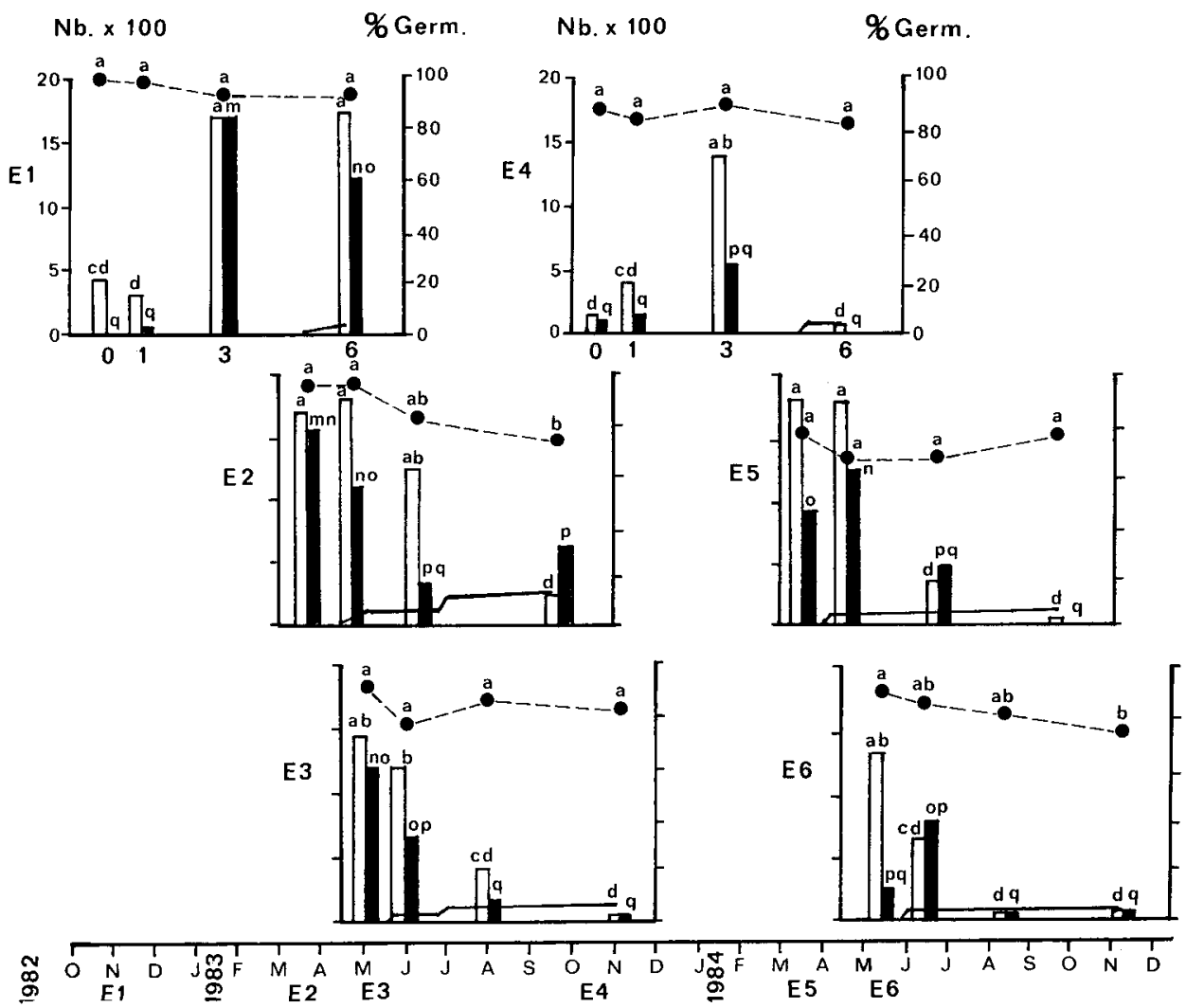
elles représentent 23 p. 100 du stock initial; en $2^{\mathfrak{C}}$ année ces extractions permettent à 6 p. 100 des semences de lever dès le mois de novembre. A la suite des extractions de printemps, les levées sont très rapides et commencent en avril-mai ; elles s'échelonnent jusqu'à octobrenovembre la $1^{\mathrm{re}}$ année pour atteindre un taux de 1824 p. 100 . En $2^{\mathrm{e}}$ année les levées sont très rapides: le taux moyen de 63 p. 100 est atteint en quelques jours et est identique au taux moyen des levées observées à la suite des extractions d'octobre 83. La courbe des levées consécutives aux extractions d'octobre 83 montre, en cours d'année, l'évolution rapide des levées : 14 jours sont suffisants pour passer de 6 à 65 p. 100 de levées, début avril. La consultation des relevés climatiques correspondant à cette période suggère que c'est l'humidité du sol, succédant à une période de sécheresse, qui provoque cette explosion de la levée des plantules; la température est alors de $7{ }^{\circ} \mathrm{C}$ en moyenne avec des amplitudes de $\pm 4^{\circ} \mathrm{C}$. Enfin, la quantité de semences retrouvées après les périodes de mise en germination au champ, est légèrement inférieure à la quantité initialement enfouie, diminuée des levées observées.

\section{DISCUSSION}

Les semences d'Aethusa cynapium sont toujours très dormantes au moment de leur dissémination (LONCHAMP \& GORA, 1980b; LONCHAMP et al., 1984 ; ROBERTS \& BODDRELL, 1985), et nous avons observé que la post-maturation au sec diminue l'aptitude à germer (tabl. 2A). Par contre lorsque les semences sont récoltées avant leur maturation morphologique (akènes jaunes ou verts), elles sont capables de germer; nous avons obtenu au laboratoire des taux de germination qui atteignaient 50 p. 100. ROBERTS \& BODDRELL (1985) constatent que l'élimination des téguments permet d'obtenir entre 25 et 75 p. 100 de germination. La difficulté de germination des semences d'Aethusa résulte donc probablement d'une inhibition tégumentaire qui se met en place en cours de maturation, se maintient et se conforte durant cette période. Par ailleurs, différentes observations sur le comportement des semences en conditions contrôlées (LONCHAMP \& MORISOT, 1984; ROBERTS \& BODDRELL, 1985) ou naturelles (LONCHAMP et al., 1984) et après action de GA3 (tabl. 2A) suggèrent une dormance propre de l'embryon.

La conservation des akènes dans un sol tassé et non travaillé sur une période de 2 ans est très bonne: Chadoeuf et al. (1980), Lonchamp et al. (1984) n'ont pas observé de disparition des semences durant de telles périodes. ROBERTS (1979), dans des sols travaillés 3 à 4 fois par an observe encore après 5 ans, 20 p. 100 des semences initialement enfouies. CHANCELlor (1964) a observé que les levées sont toujours issues de germinations superficielles, et qui n'apparaissent qu'exceptionnellement en deçà de $5 \mathrm{~cm}$ de profondeur en sol argileux. Dans notre expérimentation, la disparition des semences est donc liée essentiellement à leur germination suivie de la levée de plantules; il n'y a pas de germination en profondeur. La capacité germinative des semences évolue favorablement à la suite de l'enfouissement des semences; LONCHAMP \& MORISOT (1984)

\section{$D$ : Sinapis arvensis}

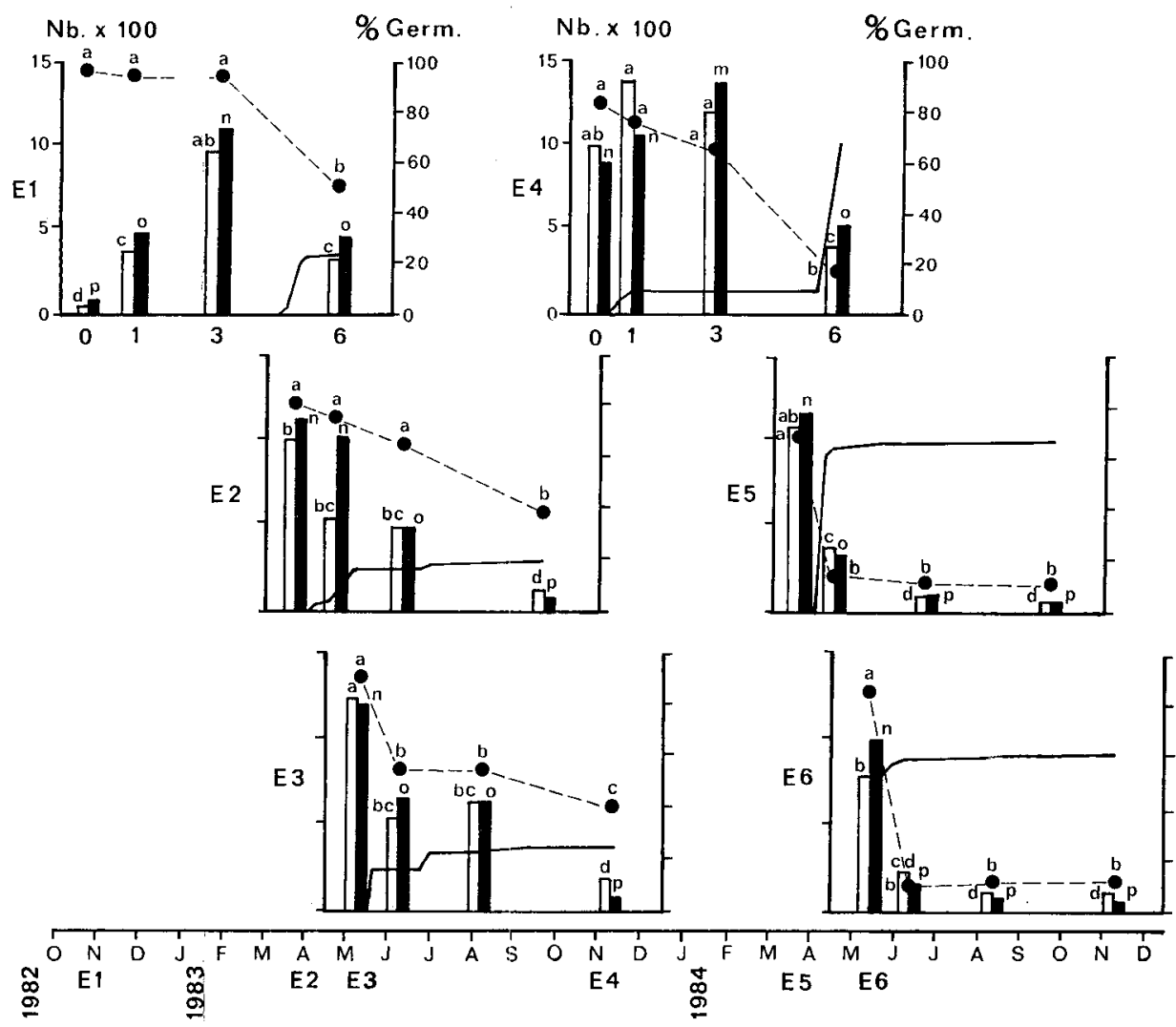


montrent qu'à $20^{\circ} \mathrm{C}, 10$ jours d'enfouissement permettent d'atteindre 9 p. 100 de germination en présence de lumière et 40 jours, plus de 50 p. 100, alors qu'à l'obscurité le taux de germination reste voisin de 0 p. 100. L'évolution cyclique de périodicité annuelle de la capacité germinative mise en évidence chez cette espèce par LONCHAMP et al. (1984) et ROBERTS \& BODDRELL (1985) explique bien le comportement des semences dans notre expérimentation: capacité germinative élevée en hiver plus importante en 1984 qu'en 1983.

La levée des plantules survient en mars-avril à la suite des extractions d'automne et jusqu'au début juin en 1984 pour les extractions tardives. Ces résultats sont comparables aux observations de LONCHAMP et al. (1984) sous cultures d'orge de printemps et de blé d'hiver. Les plus fortes levées observées en deuxième année illustrent bien la quasi-absence de dormance des semences durant l'hiver 1984.

Les semences d'Euphorbia exigua sont elles aussi très dormantes à la récolte ; cette dormance évolue favorablement avec le temps pour permettre, dans notre expérimentation, une germination en présence de lumière de 22 p. 100 après 2 ans de conservation au sec. A l'obscurité, cette évolution est peu marquée, l'acide gibbérellique est moins efficace et l'on constate que la dormance semble se renforcer au cours de la conservation.

Les semences sèches ne sont apparemment pas photosensibles; après enfouissement à -20 puis à $-1,5 \mathrm{~cm}$ on observe fréquemment l'apparition d'une photosensibilité négative. Ces résultats confirment les observations déjà réalisées par LoNCHAMP et al. (1984) chez cette même espèce. Ces auteurs montrent que, dans le sol, la capacité germinative de ces semences est corrélée étroitement avec les basses températures et la durée de l'enfouissement. On observe effectivement que les potentialités de germination s'expriment mieux de novembre à mai que pour les autres mois de l'année, surtout lors de la $2^{c}$ année d'enfouissement, plus froide que la précédente.

Le stock en semences enfouies évolue très peu en 2 ans, alors que CHADOEuf et al. (1980) constatent dans une expérience similaire que 40 p. 100 des graines ont disparu durant cette période. Après extraction des cagettes et replacement superficiel, les semences germent très peu et paradoxalement on observe le plus grand nombre de levées (13 p. 100 du stock initial) à la suite des extractions d'automne de la première année. Cette limitation dans le temps de la période de levée n'est pas en accord avec les observations de ces auteurs sur la chronologie des levées en cultures d'automne et de printemps où l'on peut observer l'apparition de plantules jusqu'à fin mai. En effet, en situation culturale normale, les semences se répartissent sur l'ensemble de la couche travaillée et subissent à diverses profondeurs des conditions d'environnement différentes permettant l'allongement de la période de germination.

La dormance des akènes de Chenopodium album est très importante, principalement à l'obscurité, et évolue peu au cours de la conservation au sec (tabl. 2C). L'acide gibbérellique permet de lever partiellement cette dormance. Williams \& HARPER (1965), et HENSON (1970) ont montré que les nitrates favorisent également la germination, tant à la lumière qu'à l'obscurité, y compris dans le sol. Cette constatation peut expliquer en partie l'évolution de la capacité germinative des semences enfouies, en relation avec l'apport de fertilisants azotés et la minéralisation de l'azote organique au printemps.

Cette espèce est connue pour l'hétérogénéité de germination de ses semences : les akènes noirs sont beaucoup plus dormants que les bruns (Williams \& HarPER, 1965; Chu et al., 1978; CAussanel, 1980). Cette hétérogénéité a été corrélée à la photopériode d'illumination des porte-graines au cours de la formation et de la maturation des semences (BASSET \& CROMPTON, 1978).

La profondeur maximale de levée est estimée par Chancellor (1964) à $4 \mathrm{~cm}$; ces levées représentent dans les meilleures conditions d'extraction (mars 83, fig. 1c), 9,4 p. $100 \mathrm{du}$ stock initial en semences. La dormance est donc très importante dans les conditions de plein champ. RoBERTS \& RICKETTS (1979) estiment que les levées de chénopodes observées dans différentes cultures sont comprises entre 0 et 6 p. $100 \mathrm{du}$ stock semencier apprécié sur $10 \mathrm{~cm}$ de profondeur. Les semences qui ne germent pas superficiellement ou qui sont enfouies à des profondeurs supérieures, se conservent dans le sol à l'état dormant : dormances innées, induites et imposées; la dormance induite semble se renforcer avec la durée d'enfouissement (tabl. 2C).

Les quelques levées observées apparaissent entre les mois d'avril et juillet, ce qui est confirmé par les observations de Chancellor (1979), Roberts \& NeILSON (1980) et Roberts \& PotTer (1980). Cependant KARSSEN (1980-1981) signale un écotype particulier à comportement d'annuelle d'hiver, germant au champ dès février. Le maximum de levées intervient normalement en juin, à une époque où la capacité germinative est relativement faible. Enfin, BASKIN \& BASKIN (1987) ont montré que les possibilités de germination étaient fonction des températures subies dans le sol par les semences enfouies après leur maturation : les akènes ne peuvent germer à basses températures qu'après stratification, ce qui exclut l'apparition de levées automnales.

Les graines de Sinapis arvensis ont un comportement très variable suivant leur provenance; le lot étudié manifeste une dormance à la récolte qui disparait progressivement au cours des premiers mois de conservation en laboratoire. JAUZEIN (1980) observe le même comportement et mentionne, comme l'ont observé LONCHAMP \& GORA $(1980, b)$, qu'une dormance peut apparaître secondairement durant la conservation au sec. La baisse d'efficacité de GA3 au cours de la conservation au sec (tabl. 2D) illustre la nature composite de la perte de l'aptitude à germer.

LONCHAMP \& GORA (1980a) ont mis en évidence sur une durée de 42 mois une évolution de l'aptitude à germer des semences de moutarde des champs : capacité germinative élevée en hiver-printemps, faible en étéautomne et d'autant plus importante que la durée d'enfouissement augmente. Les semences conservées au sec et l'enfouissement à température constante de $20^{\circ} \mathrm{C}$ (LONCHAMP \& MORISOT, 1984) augmentent la capacité germinative, on peut considérer que la réponse germinative des semences enfouies en plein champ est la résultante d'une diminution régulière de la dormance et d'une fluctuation saisonnière de celle-ci. Cependant, dans les conditions culturales normales, les semences germent et lèvent facilement après mise en germination : 
les semences dormantes constituent alors une fraction plus importante du lot initial (hétérogénéité physiologique des graines de moutarde des champs mise en évidence par JAUZEIN, 1980) qui, en l'absence de réinfestation, germeront difficilement.

Chancellor (1964) a observé que 90 p. 100 des levées de plein champ sont issues de graines enfouies à moins de $4 \mathrm{~cm}$, LONCHAMP \& GORA (1980a) estiment que le taux de disparition des semences était constant entre 5 et $20 \mathrm{~cm}$ et voisin de 12 p. 100 en 42 mois, enfin Chadoenf et al. (1980) signalent une disparition de 20 à 30 p. 100 en 1 an d'un stock semencier enfoui dans les mêmes conditions que dans notre expérimentation. Ces chiffres suggèrent que les pertes en semences par germination dans le sol, non expliquées par les levées, sont réelles : on peut les estimer dans notre étude à 24 p. 100 en 2 ans.

La chronologie des levées au champ est confirmée par les travaux de Chancellor (1979), Roberts \& Boddrell (1983) et Beuret (1984); la plupart des levées survient entre les mois de mars et juin, quelquesunes sont observées jusqu'en septembre-décembre. Cet étalement de la période de levée est vraisemblablement dû à l'hétérogénéité physiologique importante des graines chez cette espèce (JAUZEIN, 1980), que l'enfouissement, comme l'ont montré Chadoeuf-Hannel \& BARRALIS (1983) chez Amaranthus retroflexus, ne permet pas d'estomper.

La post-maturation des graines dans le sol n'a pas instauré de photosensibilité positive comme nous l'avons observé durant la conservation au sec. Wesson \& WAREING (1967) ont constaté que Sinapis arvensis est une des rares espèces de mauvaises herbes chez lesquelles l'enfouissement en plein champ n'induit pas de photosensibilité positive. Cependant, lorsque cette exigence germinative existe, LONCHAMP \& MORISOT (1984) ont montré qu'elle subsistait après un enfouissement des graines à $20^{\circ} \mathrm{C}$.

Les 4 espèces nécessitent une stratification des semences. La capacité germinative correspondant aux extractions d'octobre suivie d'un replacement superficiel de 3 mois est toujours élevée (fig. 1), ce qui suggère que la stratification est plus efficace à $1,5-2 \mathrm{~cm}$ qu'à $20 \mathrm{~cm}$ d'enfouissement. Par contre, les extractions tardives (mai) abaissent généralement la capacité de germination. A la suite de ces extractions les semences se trouvent plus longtemps exposées aux températures élevées du sol en été (principalement lors des mises en germination de 6 mois) et il est possible, comme l'a montré Courtney (1968) chez Polygonum aviculare, que ces températures induisent une dormance.

Dans cette expérimentation, l'enfouissement des cagettes et leur mise en place superficielle après extraction, sont réalisés à des profondeurs bien déterminées, et permettent d'obtenir une réponse germinative homogène : l'étalement des levées est très limité dans le temps, beaucoup plus qu'en plein champ. L'évolution de la capacité germinative est soumise alternativement au froid qui lève la dormance des semences et aux températures élevées du sol défavorables à la germination. Dans ces conditions la couverture du sol par la culture pourrait jouer un rôle et expliquer la non-concordance des observations sous culture et en sol non cultivé. Cependant les enregistrements de températures au niveau du sol ne sont très différents (plus élevées sur sol nu et capables d'induire une dormance) qu'à partir du mois de juin.

Enfin, on observe peu de destructions de semences par intervention d'agents pathogènes, saprophytes ou par germination in situ durant les 2 années d'expérimentation, alors qu'en sol normalement cultivé le taux de décroissance du stock semencier est beaucoup plus rapide (BARRALIS et al., 1988).

\section{CONCLUSION}

Dans nos conditions expérimentales, en sol tassé non travaillé, on constate que la disparition des semences est due essentiellement aux germinations qui ont donné naissance à des plantules. Il y a peu de germination in situ suivie de mort des semences, ni à 20 , ni à $1,5-2 \mathrm{~cm}$ de profondeur, sauf chez la moutarde des champs pour laquelle les semences sont peu dormantes. Les levées sont généralement faibles pour les 3 autres espèces. 1 à 37 p. 100 pour la petite ciguë, 0 à 13 p. 100 pour la petite euphorbe et 0 à 9 p. 100 pour le chénopode. Cette faible germination au champ, ainsi que la bonne conservation des semences dans le sol, expliquent la longévité de ces espèces dans les parcelles envahies non travaillées. Par contre, chez la moutarde des levées importantes (plus de 60 p. 100 du stock initial) apparaissent à la suite des travaux précoces et tardifs de printemps, épuisant rapidement le stock semencier. Cette diminution est encore accélérée par l'autodestruction des graines germant en profondeur.

Les époques d'interventions culturales simulant l'implantation des différentes cultures (hiver, printemps précoce et tardif) ont relativement peu d'influence sur le nombre total de levées des 4 espèces étudiées. On constate cependant que :

- Les interventions tardives (mai) sont défavorables à la germination d'Aethusa cynapium et d'Euphorbia exigua : les semences de petite ciguë sont dormantes à l'obscurité et celles de la petite euphorbe, moins dormantes germent difficilement aux températures enregistrées en mai-juin. Par contre, ces températures vont permettre à Sinapis arvensis et à Chenopodium album de germer : la moutarde des champs possède en effet des semences peu dormantes à cette époque (hétérogénéité physiologique importante), et le chénopode blanc est capable de germer à températures élevées.

- Les interventions précoces (octobre) favorisent la stratification des semences et la levée ultérieure des plantules. Toutes les espèces lèvent alors dès avril, plus ou moins rapidement suivant les espèces.

Sur le plan méthodologique, le contrôle précis des niveaux et des dates d'enfouissement induit une homogénéité de réponse des semences, différente de ce que l'on peut observer en plein champ. En effet, en conditions agronomiques, les semences enfouies sont d'âges différents : leurs exigences germinatives sont donc moins bien définies; de plus leur répartition dans le sol sur une profondeur de $0-25 \mathrm{~cm}$ diversifie les relations avec leur environnement et modifie l'intensité de leur dormance. 


\section{RÉFÉRENCES BIBLIOGRAPHIQUES}

Barralis G., Chadoeuf R., Lonchamp J.-P., 1988. Longévité des semences de mauvaises herbes dans un sol cultivé. Weed Research, 28.

Baskin J. M., Baskin C. C., 1987. Temperature requirements for after-ripening in buried seeds of four summer annual weeds. Weed Research, 27, 385-389.

Bassett I. J., Crompton C. W., 1978. The biology of Canadian weeds 32: Chenopodium album L. Can. J. Plant Sci., 58, 1061-1072.

Beuret E., 1984. Stocks grainiers des sols et pratiques culturales : la relation flore réelle-flore potentielle. Recherche Agron. en Suisse, 23, 89-97.

Caussanel J. P., 1980. Facteurs influençant la germination et les premiers stades de développement chez Chenopodium album L. $V I^{e}$ Coll. Int. Ecol. Biol. Syst. des Mauvaises Herbes, Montpellier, 83-94.

Chadoeuf R., Magnière J. P., Lonchamp J. P., Barralis G., 1980 Evolution comparée de la faculté germinative de semences de mauvaises herbes enfouies ou conservées au sec. $V I^{r}$ Coll. Int. Ecol. Biol. Syst. Mauvaises Herbes, Montpellier, 103-112.

Chadoeuf-Hannel R., 1985. La dormance chez les semences de mauvaises herbes. Agronomie, 5, 761-772.

Chadoeuf-Hannel R., Barralis G., 1983. Evolution de l'aptitude à germer des graines d'Amaranthus retroflexus L. récoltées dans différentes conditions, au cours de leur conservation. Weed Research, 23, 109-117.

Chancellor R. J., 1964. The depth of seed germination in the field VII' British Weed Control Conf., Brighton, 607-613.

Chancellor R. J., 1979. The seasonal emergence of dicotyledonous weed seedlings with changing temperature. Symp. Influence of Diff. factors on the Devel. and Control of weeds. Mainz, 65-72.

Chu C. C., Sweet R. D., Ozbun J. L., 1978. Some germination characteristics in Common Lambsquarters (Chenopodium album). Weed Science, 26, 255-258.

Courtney A. D., 1968. Seed dormancy and field emergence in Polygonum aviculare. J. Appl. Ecol., 5, 675-683.

Henson I. E., 1970. The effects of light, potassium nitrate and temperature on the germination of Chenopodium album $\mathbf{L}$. Weed Res. 10, 27-39.

Jauzein P., 1980. Caractéristiques physiologiques de la germination de Sinapis arvensis L. VI Coll. Int. Ecol. Biol. Syst. Mauvaises herbes, Montpellier, 63-72.

Jauzein P., 1986. Echelonnement et périodicité des levées de mauvaises herbes. Bull. Soc. bot. Fr., 133, 155-166.
Karssen C. M., 1980-1981. Patterns of change in dormancy during burial of seeds in soil. Isr. J. Bot., 29, 65-73.

Karssen C. M., 1982. Seasonal patterns of dormancy in weed seeds, 243-270. In Khan A. A.: The physiology and biochemistry of seed development, dormancy and germination. Elsevier Biomedical Press Amsterdam, New York, $547 \mathrm{p}$.

Lonchamp J.-P., Gora M., 1980a. Effet de l'enfouissement sur les exigences germinatives de mauvaises herbes. VI Coll. Int. Ecol. Biol. Syst. Mauvaises herbes, Montpellier, 113-122.

Lonchamp J.-P., Gora M., 1980b. Evolution de la faculté germinative de semences de mauvaises herbes au cours de leur conservation au sec. I.N.R.A., Lab. Malherbol., BV 1540, 21034 Dijon Cedex, 66 p.

Lonchamp J.-P., Morisot D., 1984. Effets de l'enfouissement en conditions contrôlées sur la capacité germinative de semences de mauvaises herbes. VII Coll. Inter. Ecol. Biol. et Syst. des Mauvaises Herbes, Paris, 29-35.

Lonchamp J.-P., Chadoeuf R., Barralis G., 1984. Evolution de la capacité germinative des semences de mauvaises herbes enfouies dans les sols. Agronomie, 4, 671-682.

Roberts H. A., 1979. Periodicity of seedling emergence and seed survival in some Umbelliferae. Journal of Applied Ecology, 16, 195201.

Roberts H. A., Boddrell J. E., 1983. Seed survival and periodicity of seedling emergence in eight species of Cruciferae. Ann. appl. Biol., 103, 301-309.

Roberts H. A., Boddrell J. E., 1985. Temperature requirements for germination of buried seeds of Aethusa cynapium L. Weed Research, $25,267-274$.

Roberts H. A., Neilson J. E., 1980. Seed survival and periodicity of seedling emergence in some species of Atriplex, Chenopodium, Polygonum and Rumex. Ann. appl. Biol., 94, 111-120.

Roberts H. A., Potter M. E., 1980. Emergence patterns of weed seedlings in relation to cultivation and rainfall. Weed Research, 20 , 377-386.

Roberts H. A., Ricketts M. E., 1979. Quantitative relationships between the weed flora after cultivation and the seed population in the soil. Weed Research, 19, 269-275.

Wesson G., Wareing P. F., 1967. Light requirements of buried seeds. Nature, 11, 600-601.

Williams J. T., Harper J. L., 1965. Seed polymorphism and germination. Weed Research, 5, 141-150. 